\title{
RADIO ASTRONOMY AND THE ORIGIN OF COSMIC RAYS
}

\author{
V. L. GINZBURG \\ Lebedev Physical Institute, Moscow, U.S.S.R.
}

A paper [1] sent to the Manchester Symposium on Radio Astronomy in 1955 described the views developed earlier [2] concerning a magnetobremsstrahlung (synchrotron) origin of nonthermal cosmic radio emission. Unfortunately, for unknown reasons, it was not included in the Symposium volume [3]. At the same time the paper by Unsöld was published [3] (see also [4]), in which the nonthermal galactic radio emission is connected with radiation of a great number of cold dwarfs (radio stars). At the same time, Unsöld criticizes the theory of cosmic-ray origin, according to which the main sources of these rays are envelopes of supernovae and, perhaps, novae $[2,5,6,7,8]$. Therefore, we begin with some critical remarks.

Ascribing the nonthermal cosmic radio emission to the radiation of radio stars seems inadequate for the following reasons:

1. These purely hypothetical radio stars must exist in incredibly large numbers and must have unusual properties and spatial distribution ([6], §11).

2. All identified discrete sources of cosmic radio emission are nebulae, and existence of even one radio star is not proved.

3. The emission from powerful discrete sources is doubtless of a magnetobremsstrahlung character (it is sufficient to mention the polarization of this emission); at the same time the general galactic radio emission is similar in spectrum to the radiation of discrete sources, which makes it natural to conclude that they have a common origin.

4. General galactic radio emission of a magnetobremsstrahlung origin must surely exist, since the Galaxy contains magnetic fields and relativistic electrons, from supernova envelopes, formed in the Galaxy by $\pi \rightarrow \mu \rightarrow e$ decay following collisions of cosmic-ray protons and nuclei; calculations show $[1,2,5,6,7]$ that the abovementioned magnetobremsstrahlung radiation can have the intensity and spectrum of the observed radio emission.

5. Data on the strong extragalactic discrete sources and the results of polarization measurements on the total galactic radio emission $[9,10]$ also speak in favor of the magnetobremsstrahlung origin of cosmic radio emission.

All these considerations, in our opinion, make it indubitable that the main part of the nonthermal cosmic radio emission originates by magnetobremsstrahlung and is not generated in stellar atmospheres.

It is probable that the role of stars other than supernovae and novae in the formation of cosmic rays is also small. On the average $10^{21}$ to $10^{22}$ ergs/second are converted into cosmic rays formed on the sun. Therefore all $10^{11}$ Galaxy stars, if they emitted cosmic rays like the sun, would give 
$10^{32}$ to $10^{33} \mathrm{ergs} / \mathrm{second}$. At the same time the power of the cosmic ray sources in the Galaxy must be $U \sim W / T \sim 10^{39}$ to $10^{40} \mathrm{ergs} / \mathrm{second}$, since the total energy $W$ of cosmic rays is about $10^{55}$ to $10^{58} \mathrm{ergs}$, and their mean lifetime $T$ is about $10^{\circ}$ years (we assume that the average gas concentration $n$ in the region occupied by cosmic rays is about $0.03 \mathrm{~cm}^{-3}$ ). Estimates of the average power transferred into cosmic rays as the result of outbursts of supernovae and novae lead to values of the order of $10^{39}$ to $10^{41} \mathrm{ergs} / \mathrm{second}$ (for more details see $[2,5,7,8]$ ). This estimate is based on observation and shows that supernovae and novae are really able to provide the energy balance. In order to obtain the same energy from the stars, it is necessary, for example, to have $10^{11}$ stars which are $10^{8}$ to $10^{7}$ times more active than the sun. It seems unlikely that even the magnetic stars of type A [11] satisfy such requirements. Furthermore, if the stars of well-known types made a noticeable contribution to the cosmic ray intensity, which is not proved, this fact would not speak in favor of the radio-star origin of the galactic radio emission (it is sufficient to point out the difference in spatial distribution of A-type stars and stars of other types from the distribution of the sources of general galactic radio emission).

For these reasons the present theory of origin of cosmic radio emission and cosmic rays $[1,2,5,6,7,8]$ seems to be adequately justified. However, some further points to be cleared up will be considered below.

A rather important parameter of the theory is the lifetime of cosmic rays as determined by nuclear collisions and particle exit from the Galaxy (we consider a quasi-spherical "corona" with radius $R \sim 5 \times 10^{22} \mathrm{~cm}$ filled with cosmic rays). According to [12] the average interstellar gas concentration in the Galaxy is $n \sim 10^{-2} \mathrm{~cm}^{-3}$. This corresponds to the nuclear lifetime of fast protons $T \sim 4 \times 10^{9}$ years. But the radio-astronomical data speak rather in favor* of the values $n \sim 0.03$ and $T \sim 10^{9}$ years; in this case the nuclear lifetime for $\mathrm{Fe}$ nuclei is of the order of $T_{\mathrm{Fe}} \sim 5 \times 10^{7}$ years. This time is considerably shorter than the age of the Galaxy. At the same time a rather great number of $\mathrm{Fe}$ nuclei occur in the primary cosmic rays, and there is no reason to assume that fast nuclei and protons are of a different origin. Therefore it is possible [7] to neglect the cosmic rays formed under conditions differing essentially from the present. This conclusion can be quite valid also for the density $n \leq 10^{-2}$ since the proton lifetime, in particular at low density, may be determined by exit from the Galaxy. Indeed, the estimates show [7] that with a galactic "boundary" transparency $\rho \sim 10^{-2}$, the exit lifetime $T_{0} \sim 4 \times 10^{9}$ to $4 \times 10^{10}$ years. The corresponding calculation is, however, considerably less exact than the calculation of the nuclear

* According to [13] the galactic radio emission spectrum has a "knee" at $v \simeq 10^{7} \mathrm{c} / \mathrm{s}$, which can be connected with the fact that at $v \simeq 10^{7}$ the magnetobremsstrahlung losses are equal to the ionization losses. Hence (see [7]) $n \sim 10^{3} H_{\perp \vee} \sim 0.03$ at $H_{\perp} \sim 3 \times 10^{-6}$ oersteds $\left(H_{\perp}\right.$ is the component of the interstellar magnetic field perpendicular to the particle velocity). One of the urgent problems is how to determine the spectrum of long-wave radio emission for different directions (the artificial earth satellite can be rather useful for this purpose [21]). 
lifetime. In particular, the value $\rho \sim 10^{-2}$ corresponds to the assumption that the extragalactic magnetic field is about $10^{2}$ times weaker than the galactic one, and there is some "boundary" between these regions (in other words, one assumes that only one per cent of all lines of force leave the Galaxy).*

Assuming larger values of $\rho$ and also varying other parameters, one can have exit times $T_{0} \sim 10^{9}$ years. At the same time, apparently, the effective particle lifetime $T$ determined by the relation $1 / T=1 / T_{p}+1 / T_{0}$ will be also of the order of $10^{9}$ years.

The question of fast particles of metagalactic origin is closely connected with the cosmic-ray exit from the Galaxy. In a field $H \sim 10^{-5}$, for protons with energy $E \sim 10^{18}$ electron volts, the radius of curvature $r=E / 300 \mathrm{H} \sim$ $3 \times 10^{20} \ll R \sim 5 \times 10^{22}$ (for nuclei the radius $r$ is $Z$ times less; at the same time it is not excluded that the particles with the greatest energies are nuclei). Therefore, even the exit of particles with $E \sim 10^{18}$ electron volts is, perhaps, relatively small if drift in an inhomogeneous magnetic field does not play the main role. The drift velocity $V_{d} \sim(r|\nabla H| / H) v_{\perp} \sim r v_{\perp} / L$, where $L$ is the characteristic distance for field changes and $v_{\perp}$ is the component of the particle velocity normal to the field. The number of particles leaving the Galaxy per second owing to the drift is equal to $S_{o d} \sim 4 \pi R^{2} N v_{d} \xi \sim(4 \pi / 3) 10^{-2} \xi R^{2} N c E / H L$, where the factor $\xi<1$; its appearance is connected with the fact that the drift takes place normally to the field gradient and the field itself; owing to this fact, the flux $S_{o d}$ can be considerably less than the value $4 \pi R^{2} N v_{d}$. Owing to the collisions, $S_{p} \sim(4 \pi / 3) R^{3} N / T_{p}$ particles/second vanishes, where $N$ is the concentration of fast particles. Hence

$$
\alpha=\frac{S_{o d}}{S_{p}} \sim 10^{-2} \xi \frac{c T_{p} E}{H L R} \sim \frac{10^{7} \xi E}{L},
$$

where $T_{p} \sim 10^{9}$ years $\sim 3 \times 10^{16}$ seconds, $H \sim 10^{-5}$ and energy $E$ is expressed in electron volts. One obtains the maximum value of $\alpha_{\max } \sim\left(10^{-13}\right.$ to $\left.10^{-14}\right) E$ at $\xi \sim 1$ and $L \sim l \sim 10^{20}$ to $10^{21}$, where $l$ is the characteristic size of the magnetic field inhomogeneities in the Galaxy. On the other hand, for the Galaxy as a whole, $L \sim 1^{22}$ to $10^{23}$, and at $\xi \sim 10^{-2}$ one obtains $\alpha \sim$ $\left(10^{-17}\right.$ to $\left.10^{-18}\right) E$. If one assumes the value $\alpha_{\max }$, then for energies $E>E_{k, \min } \sim$ $10^{13}$ to $10^{14}$ electron volts, particles escape from the Galaxy, and with more ease as their energy increases. ${ }^{\dagger}$ This will lead, in all probability, to a rather sharp decrease in the energy spectra for cosmic rays of galactic origin for $E>E_{k}$. Since such sharp decrease is not found in practice, one can assume that cosmic rays of metagalactic origin play an important role in the region $E>E_{k}{ }^{\ddagger}$ In this case, however, it would be natural to expect, at $E \sim E_{k}$,

* In the early stages of galactic development, the field could be weaker, and the coefficient of boundary transparency would be higher than now. If so, the role of protons formed in the early stage would be still less.

$\dagger$ Note that ease of particle exit from the Galaxy shows also ease of penetration from without, and that on the other hand if exit is difficult, entry is difficult.

$\ddagger$ This assumption is reasonable, of course, only if $E_{k}<10^{16}$ to $10^{17}$, since at large energies the spectrum is not known at all. 
some "knee" in the energy spectrum of cosmic rays (this knee will fail to show only if the rays of galactic and metagalactic origin have absolutely equal spectra). As far as is known, the spectrum has no such knee, but it is difficult here to make a final conclusion without additional measurements. In order to clear up the possible role of the metagalactic component of cosmic rays, it is important to establish, in addition to details of the spectrum, a chemical composition and the rate of cosmic ray anisotropy for energies $E>10^{13}$ to $10^{14}$ electron volts.

Another important problem of the theory of cosmic ray origin is the acceleration mechanism in the envelopes of supernovae and novae and, perhaps, in some regions of interstellar space. The general features of this problem seem to be clear (for example, there are no doubts concerning efficiency of the statistical acceleration mechanism in the envelopes, etc.). However, a whole number of substantial problems require to be cleared up or considered in more detail. Fortunately, in the scheme based on the observational data, the problem of the acceleration mechanism does not much depend on the others (see [7]). Therefore we will only mention two new aspects. Arguments have been given for the assumption [14] that the statistical acceleration in magnetohydrodynamic waves is more effective than in the model of "magnetic clouds." In other work [15] it has been shown that the statistical acceleration of nuclei with $Z>2$ can be rather effective in comparison with proton acceleration (we refer to injection conditions). This fact can play a considerable role in the analysis of the chemical composition of cosmic rays [7] (such analysis is rather difficult yet owing to the lack of sufficiently complete data on composition of the primary cosmic rays and probability of nuclear transmutations in the interstellar medium).

The third problem to be pointed out here concerns the origin of the electrons producing the total galactic radio emission. The total emission in the Galaxy is equal to $10^{38} \mathrm{ergs} / \mathrm{second}$. But protons lose one to two orders more (see above). Therefore one can assume that all replenishment of the electron component takes place by secondary processes (i.e., by $\mu^{ \pm} \rightarrow e^{ \pm}$decay of $\pi^{ \pm}$mesons formed by nuclear collisions) [5, 7].

On the other hand, supernovae and novae are also able to provide the energy for electrons, since they transfer energy of the order of $10^{37}$ to $10^{39}$ ergs/second to electrons. Thus, on the basis of energy considerations only, it is impossible to determine whether electrons in interstellar space are on the whole primary or secondary. In the main, this problem can be cleared up by investigation of spectral dependence on galactic coordinates, or of the radio brightness distribution at different frequencies in extragalactic nebulae (e.g., in the nebula M31)[16, 17, 18]. The point is that, during the course of diffusion in interstellar magnetic fields, electrons lose their energy; therefore fast electrons generated in the central regions of the Galaxy must be softer on its periphery. This leads to transformation of the spectrum of their radio emission. On the other hand, secondary electrons produced by nuclear collisions will have nearly the same spectrum everywhere. Such differences in the spectrum are not, however, very sharp, calculations include some inexact 
parameters $[16,18]$, and finally there are no observational data. For all these reasons it is impossible as yet to draw conclusions. The same can be said concerning the assumptions, on the basis of similarity of the spectra of the general galactic radio emission and the emission of the majority of discrete sources [19], that electrons have a secondary origin (for new data on the spectra of sources see [20]). If electrons in supernova envelopes and in interstellar space are generated in the first stages of the outburst, similarity of the spectra is quite natural. On the contrary, if electrons leave the envelope only in its late stages with the spectrum $N_{0}(E)=K_{0} E^{-\gamma_{0}}$ we will have another picture. After a sufficiently long journey in interstellar space, electrons will have the spectrum $N(E)=K E^{-\gamma}$, where $\gamma=\gamma_{0}+1$. In a general case, when the particle emission from the envelopes takes place continually, we will have, naturally, intermediate results. It should also be taken into account that the field strengths in the envelopes and in interstellar space are different. Owing to this fact electrons of different energies will be responsible in the two cases for the emission of a given frequency. According to the above it still seems to be impossible to conclude that the electrons in the Galaxy have predominantly a secondary origin. But, of course, the possibility is a real one. One can hope that further radio-astronomical observations will solve the problem (we mean the investigation of spectra over a wide range of frequencies and directions). Another way [5] of solving this problem is connected with the fact that the secondary light particles consist of electrons and positrons in nearly the same quantity. Therefore absence of a noticeable quantity of positrons in the light component of primary cosmic rays would speak in favor of acceleration of electrons in the sources themselves. The investigation of the light (electron-positron) component of primary cosmic rays near the earth is very important for other reasons also (a direct measurement of electron concentration would make substantially more exact the estimate of this quantity derived from the radio-astronomical data).

\section{REFERENCES}

[1] Ginzburg, V. L. "The nature of cosmic radio emission and the origin of cosmic rays," 1955, paper presented at the Manchester Symposium on Radio Astronomy by Dr. S. B. Pikel'ner.

[2] Ginzburg, V. L. Usp. Fiz. Nauk, 51, 343, 1953. Fortschr. Phys., Berlin 1, 659, 1954.

[3] Radio Astronomy (I.A.U. Symposium No. 4, 1955). Cambridge, England, 1957.

[4] Unsöld, A. Z. Phys. 141, 70, 1955.

[5] Ginzburg, V. L. Nuovo Cim., Suppl., 3, 38, 1956.

[6] Shklovskii, I. S. Cosmic Radio Emission. Moscow, 1956.

[7] Ginzburg, V. L. Origin of Cosmic Rays (Vol. IV, "Progress in ElementaryParticle and Cosmic-Ray Physics"). Amsterdam, 1958.

[8] Ginzburg, V. L. Nuovo Cim., Suppl. 8, 430, 1958.

[9] Razin, V. A. A. Zh. 35, 241, 1956.

[10] Thompson, I. M. Nature, 180, 495, 1957.

[11] Babcock, H. Phys. Rev. 109, 2210, 1958. 
[12] Pikel'ner, S. B., and Shklovskii, I. S. A. Zh. 34, 145, 1957.

[13] Ellis, G. R. J. Geophys. Res. 62, 229, 1957.

[14] Parker, E. N. Phys. Rev. 109, 1328, 1958.

[15] Korchak, A. A., and Syrovatskii, S. I. Dokl. Akad. Nauk S.S.S.R. 122, 792, 1958.

[16] Getmantsev, G.G. Transactions of the Fifth Conference on Cosmogonical Questions. Moscow, 1956, p. 468.

[17] Ginzburg, V. L. Transactions of the Fifth Conference on Cosmogonical Questions. Moscow, 1956, p. 438.

[18] Syrovatskii, S. I. A. Zh. (In press.)

[19] Shklovskii, I. S. Nuovo Cim.,Suppl. 8, 421, 1958.

[20] Whitfield, G. R. M.N.R.A.S. 117, 680, 1957.

[21] Getmantsev, G. G., Ginzburg, V. L., and Shklovskii, I. S. Usp. Fiz. Nauk, 66, $157,1958$.

\section{Discussion}

G. R. Burbidge: I would like to point out in connection with Ginzburg's paper that another observational test can be applied to decide whether the radio electrons are of primary or secondary origin. If they are secondary then it may be possible to detect strong radio sources as gamma-ray sources.

Savedoff: In extension of Dr. Burbidge's remarks, observations of $\pi^{0}$ by its decay into gamma rays of, for the Crab, an estimated flux of $10^{-4}$ photons $\mathrm{cm}^{-2} \mathrm{sec}^{-1}$ may be possible. Gamma rays from the equally numerous $\pi^{+}$and $\pi^{-}$will give an equal flux concentrated in a relatively narrow line produced by the $e^{+}$and $e^{-}$annihilation. This latter is probably easier to observe. The University of Rochester cosmic-ray group is beginning experimental work which we hope will show whether this is observable at balloon altitudes. 